\title{
Morning/Evening Differences in Somatosensory Inputs for Postural Control
}

\author{
Clément Bougard ${ }^{1,2,3}$ and Damien Davenne $e^{3,4,5}$ \\ ${ }^{1}$ Armed Forces Biomedical Research (IRBA), Vigilance Team, 91223 Brétigny-sur-Orge, France \\ ${ }^{2}$ Université Paris Descartes, Sorbonne Paris Cité, EA 7330 VIFASOM Sommeil-Fatigue-Vigilance et Santé Publique, 75181 Paris, France \\ ${ }^{3}$ Normandie University, 14032 Caen, France \\ ${ }^{4}$ Unicaen, COMETE, 14032 Caen, France \\ ${ }^{5}$ INSERM, U 1075, COMETE, 14032 Caen, France
}

Correspondence should be addressed to Clément Bougard; clement.bougard@irba.fr

Received 15 March 2014; Revised 24 July 2014; Accepted 24 July 2014; Published 18 August 2014

Academic Editor: Jacob J. Sosnoff

Copyright (C) 2014 C. Bougard and D. Davenne. This is an open access article distributed under the Creative Commons Attribution License, which permits unrestricted use, distribution, and reproduction in any medium, provided the original work is properly cited.

\begin{abstract}
The underlying processes responsible for the differences between morning and afternoon measurements of postural control have not yet been clearly identified. This study was conducted to specify the role played by vestibular, visual, and somatosensory inputs in postural balance and their link with the diurnal fluctuations of body temperature and vigilance level. Nineteen healthy male subjects (mean age: $20.5 \pm 1.3$ years) participated in test sessions at 6:00 a.m. and 6:00 p.m. after a normal night's sleep. Temperature was measured before the subjects completed a sign cancellation test and a postural control evaluation with eyes both open and closed. Our results confirmed that postural control improved throughout the day according to the circadian rhythm of body temperature and sleepiness/vigilance. The path length as a function of surface ratio increased between 6:00 a.m. and 6:00 p.m. This is due to a decrease in the centre-of-pressure surface area, which is associated with an increase in path length. Romberg's index did not change throughout the day; however, the spectral analysis (fast Fourier transform) of the centre-of-pressure excursions (in anteroposterior and mediolateral directions) indicated that diurnal fluctuations in postural control may occur via changes in the different processes responsible for readjustment via muscle contractions.
\end{abstract}

\section{Introduction}

Various studies reported an influence of time-of-day on postural control [1-5], even if the results were founded on various materials, experimental procedures, and evaluation criteria [6]. When external parameters were controlled, it has been shown that the greatest variations in balance capacities throughout a normal day were observed between 6:00 a.m. and 6:00 p.m. [7]. However, the underlying processes responsible for these diurnal fluctuations in postural control have not yet been clearly identified.

Balance is maintained by the continuous and effective integration of vestibular, visual, and proprioceptive information in the central nervous system (CNS) [8]. All of this sensorial information is processed in the cerebellum, enabling the centre of gravity (CG) to be supported and maintained by postural muscle contractions [9]. More precisely, the vestibulocerebellum is a cerebral structure that is involved in postural control regulation [10] and visuomotor coordination [11]. Another part of the cerebellum, called the cerebrocerebellum, is involved in the regulation of various nonmotor functions such as attention and/or cognition [12]. Moreover, various studies reported changes in cerebellum activation during sleep [13] and with increased sleepiness [14], which can impact motor activity. As a consequence, a number of studies suggested that close relationships between balance capacities and the level of sleepiness can be considered $[1,4$, $6,7]$.

Through the use of spectral analysis [9], the evaluation of the possible contributions of vestibular, visual, and somatosensory inputs to postural control throughout the day is of great interest. Even if there is still debate on this 
approach, either on the range of total frequency content $[15,16]$ or band-width size $[15,16]$, studies based on sleep deprivation paradigms (which modify the level of sleepiness/vigilance) reported that the vestibular system would be the sensory input most affected by an increase in the length of time awake $[2,4,6]$. But other studies concluded that visual input becomes less efficient with the length of time awake, or that the integration of visual information becomes deficient or slower [17].

While the postural control system appears to use distinct control strategies in the anteroposterior and mediolateral directions [18], the spectral analysis was only conducted on the global signal. Recent studies have shown that the identification of somatosensory inputs has to take into account the spectral content of postural sway on each direction separately (anteroposterior and mediolateral) $[19,20]$. This is because the evolution of one sensory input can be different in each direction throughout the day. This approach can give further information on the underlying processes responsible for the previously observed changes in postural strategies by using the distribution of variability at different frequencies. While investigating time-of-day effects on postural control, one would expect that postural sway in the anteroposterior and mediolateral directions may require different processes in the morning than in the afternoon.

Furthermore, it would be interesting to test the circadian rhythms of other physiological variables such as temperature and vigilance (which are the most studied because of their repercussions on human behaviour [21]) to examine if they evolve similarly to postural control fluctuations. This would notably bring further information on possible compensatory adjustments. Temperature is often used as the gold standard test to evaluate circadian rhythmicity [21]. Various studies have shown that postural sway evolves in harmony with the temperature rhythm [22, 23], peaking in the early morning (between 5:00 a.m. and 7:00 a.m.), during the bathyphase of the body temperature rhythm [2, 22]. Temperature also influences many other physiological regulations that are involved in postural sway regulations [24]. Vigilance reflects the level of CNSs activation and the varied levels can be estimated on a continuum between wake and sleep (vice versa for sleepiness) [25]. It has been shown that postural control is modified by the level of vigilance or sleepiness $[4,6,17,22,23,26-28]$. Since maintaining balance requires continuous integration of different sensory inputs, it has been proposed that vigilance impairment affects the processes of these integrations at the CNS level and, in parallel, also affects the processes underlying efficient adjustments of movements involved in postural sway regulation $[17,26,27]$.

In this study, oral temperature, vigilance, and postural sway parameters have been recorded in parallel to specify (i) the link between these parameters and (ii) the role played by vestibular, visual, and somatosensory inputs in the diurnal fluctuations of postural control. For that, a frequency analysis applied on centre-of-pressure excursions has been used in the anteroposterior and mediolateral directions.

\section{Methods and Materials}

2.1. Subjects. Nineteen nonsmoking male subjects (age: $20.5 \pm$ 1.3 years; body mass: $70.0 \pm 6.1 \mathrm{~kg}$; height: $179.0 \pm 4.8 \mathrm{~cm}$ ) participated in this study, which was granted ethical approval by the ethics committee (Comité de Protection des Personnes Nord-Ouest III, number 2007-A00581-52), and has therefore been performed in accordance with the ethical standards established in the 1964 Declaration of Helsinki. After being informed of the various procedures and objectives of the study, all subjects signed a consent form. The subjects were selected according to their absence of excessive diurnal sleepiness measured by the Epworth Sleepiness Scale (score < 10) [29] and their chronotype established on the basis of their answers to the Horne and Ostberg [30] questionnaire. Only the following chronotypes were included in the study: neither morning nor evening $(n=11)$, moderately morning $(n=2)$, and moderately evening $(n=6)$ types. Subjects who were definitively morning or definitively evening types were excluded $(n=5)$ as their circadian rhythms may have been phase-advanced or phase-delayed in comparison to moderate morning or evening types [31]. Therefore, as a result, they may have induced large interindividual variability in the results [32].

2.2. Procedure. The subjects took part in two experimental test sessions set up at 6:00 a.m. and 6:00 p.m. in a counterbalanced order. When the subjects were due to take part in the morning test session, they were gathered in the laboratory the night before from 9:30 p.m. to lie down to sleep at 10:30 p.m. The subjects were woken up at 5:00 a.m. in order not only to guarantee a minimum of $6 \mathrm{~h}$ in bed, but also to respect a $1 \mathrm{~h}$ waking period before the test session $[33,34]$. These precautions are generally applied in chronobiological studies in order to avoid the effects of partial sleep deprivation [35] and sleep inertia [36] on measurements carried out in the morning. In addition, no food or drink was consumed before this test session in order to limit interindividual variability observed in cognitive, psychomotor, and physical performances $[33,34]$.

When the subjects were evaluated at 6:00 p.m., they were instructed to consume the preceding meal at least $3 \mathrm{~h}$ before the test session [32] and to come to the laboratory at 3:00 p.m. The consumption of stimulant drinks (coffee, tea, and energy drinks) and participation in physical activities were prohibited in order to avoid their masking effects on diurnal fluctuations in postural control [37]. The mean ambient temperature of the laboratory was $21.9+1.8^{\circ} \mathrm{C}$ during the test sessions.

\subsection{Measurements}

2.3.1. Temperature. Before each test session the subjects were asked to lie down and relax and not to eat or drink anything for $15 \mathrm{~min}$ [38]. Then the oral temperature was measured by an experimenter using a digital clinical thermometer (Omron, accuracy: $0.05^{\circ} \mathrm{C}$ ), inserted sublingually for at least $3 \mathrm{~min}$. 


\subsubsection{Sleepiness/Vigilance}

Sign Cancellation Test. Vigilance was evaluated by a sign cancellation test [39]. A sheet of paper with 25 lines made up of 20 signs, each distributed at random (i.e., eight different forms for a total of 500 signs), was given to each subject. After sitting at a table in a quiet and neutral room, each subject was instructed to cross out all of the signs that matched three of the eight types present. The three shapes to be crossed out were changed in each test session. Various performance indicators were selected, such as time taken to complete the grid and the total number of errors, which corresponded to both omissions and false alarms (i.e., signs that were incorrectly crossed out). This type of test has been selected because it is particularly sensitive in revealing the effects of time-of-day on vigilance [40].

2.3.3. Posturographic Assessments. The capacity of the subjects to maintain their balance was evaluated using a force platform (PostureWin (), Techno Concept, Céreste, France; $40-\mathrm{Hz}$ frequency, 12-bit A/D conversion) that recorded displacements in the centre of foot pressure (COP) with three strain gauges. The subjects were placed according to precise marks. Their legs were stretched out and their feet formed a $30^{\circ}$ angle relative to each other (intermalleolar distance of $5 \mathrm{~cm}$ ). The test lasted $51.2 \mathrm{~s}$ and was first performed with eyes open (EO) and then with eyes closed (EC). In the EO condition, the subjects looked at a fixed level target $90 \mathrm{~cm}$ away. In the EC condition, they were asked to keep their gaze straight-ahead without speaking or clenching their teeth.

The COP surface area (90\% confidence ellipse) evaluates a subject's postural performance: the smaller the area, the better the performance [41]. The path length (PL), the anteroposterior PL, the mediolateral PL, and the ratio corresponding to the length of the COP displacement according to the surface (LFS: length as a function of surface), index of the energy expenditure [42], and Romberg's index (RI) ((surface $\mathrm{EC} /$ surface EO) $\times 100)$, which evaluates the contribution of vision to the maintenance of posture [43], were also computed.

Fast Fourier transforms were applied to the COP displacements from 0 to $20 \mathrm{~Hz}$. Hence, the total spectral energy was calculated and distributed between three frequency bands: low frequencies (LF): $0-0.5 \mathrm{~Hz}$; medium frequencies (MF): 0.5-2 Hz; and high frequencies (HF): $>2 \mathrm{~Hz}$ [44], for the anteroposterior and mediolateral directions [19]. The values of these three frequency bands were expressed as a percentage of the total spectral energy [45]. Low frequencies mostly account for visual and vestibular regulation $[1,46]$, medium frequencies for cerebellar regulation [45], and high frequencies for the involvement of the reflexive loop [20, 44, 46].

2.4. Statistical Analysis. In order to determine possible differences between the data obtained at 6:00 a.m. and at 6:00 p.m., oral temperature and the time to complete the sign cancellation test were analysed using a $t$-test for matched samples.
The total number of errors, the number of errors made by omission, and the number of false alarms that occurred during the sign cancellation test provided quantitative and discontinuous data. A Wilcoxon test for matched samples was applied to the results obtained at 6:00 a.m. and at 6:00 p.m.

Postural sways were analysed by a 2 (time-of-day: 6:00 a.m. versus 6:00 p.m.) $\times 2$ (condition of vision: normal versus occluded) repeated-measure analysis of variance (ANOVA). Dependent variables were COP surface area, PL, anteroposterior PL, mediolateral PL, and LFS ratio. In addition, the percentage of low-frequency, medium-frequency, and high-frequency bands obtained in the anteroposterior and mediolateral directions during the measurements with eyes open were analysed using a 2 (time-of-day: 6:00 a.m. versus 6:00 p.m.) $\times 3$ (frequency band: low, medium, and high) ANOVA. When an interaction effect was observed, a post hoc analysis (least significant difference) was applied. The total spectral energy was measured in the anteroposterior and mediolateral directions separately and Romberg's index was also calculated. The data obtained at 6:00 a.m. were compared to those obtained at 6:00 p.m. using a $t$-test for matched samples.

All differences were regarded as significant at a $P<0.05$. All statistical analyses were performed using STATISTICA $^{(}$ software (Statsoft, France, version 7.1).

\section{Results}

3.1. Oral Temperature. A significant effect of time-of-day was found for oral temperature $(t=9.55 ; P<0.01)$. The temperature was higher at 6:00 p.m. than at 6:00 a.m. $\left(+0.89^{\circ} \mathrm{C}\right)($ Table 1$)$.

3.2. Sleepiness/Vigilance. The subjects were more efficient at the sign cancellation test at 6:00 p.m. than at 6:00 p.m. $(t=$ $3.35 ; P<0.01)$ while no significant difference was observed concerning the errors made by omission ( $z=1.44$; NS) or false alarms $(z=0.59$; NS) according to the time-of-day (Table 1).

3.3. Posturographic Assessments. A main effect of the condition of vision was observed on all parameters $\left(\mathrm{PL}\left(F_{(1,18)}=\right.\right.$ 133.4; $P<0.001)$, anteroposterior PL $\left(F_{(1,18)}=116.4 ; P<\right.$ 0.001), mediolateral PL $\left(F_{(1,18)}=30.57 ; P<0.001\right)$, COP surface area $\left(F_{(1,18)}=22.04 ; P<0.001\right)$, and LFS ratio $\left(F_{(1,18)}\right.$ $=6.55 ; P<0.05))$. These parameters were all increased under the EC condition in comparison with the EO condition (Table 1).

The ANOVA indicated a significant effect of time-of-day on COP surface area $\left(F_{(1,18)}=11.84 ; P<0.01\right)$. Regardless of the condition of vision, the COP surface area decreased by $27.5 \%$ in the evening compared to the morning values. In addition, time-of-day also had a significant effect on the LFS ratio $\left(F_{(1,18)}=5.02 ; P<0.05\right)$. The average LFS ratio was higher in the evening $(+8.4 \%)$ compared to the morning values (Table 1).

More importantly, an interaction effect of "time-of-day" $\times$ "condition of vision" was observed on PL $\left(F_{(1,18)}=5.90\right.$; $P<0.05)$, mediolateral PL $\left(F_{(1,18)}=4.74 ; P<0.05\right)$, and 
TABLE 1: Oral temperature, sign cancellation test, and posturography parameters in subjects under eyes open (EO) and eyes closed (EC) conditions at 6:00 a.m. and 6:00 p.m. (mean \pm SD; $n=19$ ).

\begin{tabular}{|c|c|c|c|}
\hline & & 6:00 a.m. & 6:00 p.m. \\
\hline Oral temperature $\left({ }^{\circ} \mathrm{C}\right)$ & & $35.99 \pm 0.32$ & $36.77 \pm 0.22^{*}$ \\
\hline Sign cancellation test (s.) & & $340.36 \pm 62.46$ & $287.01 \pm 53.41^{*}$ \\
\hline \multirow{2}{*}{ COP surface area $\left(\mathrm{mm}^{2}\right)$} & $\mathrm{EO}$ & $120.73 \pm 62.89$ & $89.03 \pm 40.21^{*}$ \\
\hline & $\mathrm{EC}$ & $179.76 \pm 86.52$ & $128.89 \pm 60.43^{*}$ \\
\hline \multirow{2}{*}{ PL (mm) } & $\mathrm{EO}$ & $375.28 \pm 117.34$ & $423.49 \pm 107.28^{*}$ \\
\hline & $\mathrm{EC}$ & $539.86 \pm 143.57$ & $539.28 \pm 149.04$ \\
\hline \multirow{2}{*}{ Mediolateral PL (mm) } & $\mathrm{EO}$ & $188.23 \pm 63.09$ & $208.91 \pm 54.59^{*}$ \\
\hline & EC & $251.64 \pm 78.33$ & $247.95 \pm 68.87$ \\
\hline \multirow{2}{*}{ Anteroposterior PL (mm) } & $\mathrm{EO}$ & $284.25 \pm 90.96$ & $316.07 \pm 88.32$ \\
\hline & EC & $424.58 \pm 114.18$ & $420.51 \pm 130.14$ \\
\hline \multirow{2}{*}{ LFS ratio } & $\mathrm{EO}$ & $0.86 \pm 0.28$ & $0.98 \pm 0.22^{*}$ \\
\hline & EC & $0.96 \pm 0.26$ & $0.99 \pm 0.24$ \\
\hline Romberg's index & & $168.54 \pm 84.14$ & $150.67 \pm 50.46$ \\
\hline
\end{tabular}

COP surface area: centre-of-pressure surface area; PL: path length; anteroposterior PL: anteroposterior path length; lateral PL: lateral path length; LFS ratio: path length as a function of surface; Romberg's index: ratio between the centre-of-pressure surface areas measured under the eyes open (EO) and eyes closed (EC) conditions. ${ }^{*}$ Significant difference between 6:00 a.m. and 6:00 p.m.

LFS ratio $\left(F_{(1,18)}=5.70 ; P<0.05\right)$. The post hoc analysis indicated that, under the EO condition, PL, mediolateral PL, and the LFS ratio increased from 6:00 a.m. to 6:00 p.m. by $12.8 \%, 10.9 \%$, and $14.7 \%$, respectively. However, these three parameters were not modified throughout the day under the EC condition. Moreover, it must be noted that Romberg's index did not change throughout the day $(t=0.85$; NS).

The analysis of the total spectral energy in the anteroposterior direction based on the fast Fourier transform showed no significant difference ( $t=-1.66$; NS) between 6:00 a.m. $\left(17.20 \pm 4.52 \mathrm{~mm}^{2} \cdot \mathrm{Hz}^{-1}\right)$ and $6: 00$ p.m. (18.85 \pm $\left.4.47 \mathrm{~mm}^{2} \cdot \mathrm{Hz}^{-1}\right)$. More precisely, the statistical analysis indicated that in the anteroposterior direction the contribution of the low-frequency band (52.9\%) was higher than that of the medium-frequency (30.1\%) and high-frequency bands $(16.9 \%)$, regardless of the time-of-day $\left(F_{(2,36)}=128.19 ; P<\right.$ $0.001)$. Moreover, an interaction effect of "time-of-day" $\times$ "frequency band" was observed $\left(F_{(2,36)}=9.45 ; P<0.001\right)$. The proportion of the low-frequency band decreased $(9.61 \pm$ $2.81 \mathrm{~mm}^{2} \cdot \mathrm{Hz}^{-1}$ (i.e., $56.12 \pm 8.86 \%$ ) at $6: 00$ a.m. versus $9.36 \pm$ $2.39 \mathrm{~mm}^{2} \cdot \mathrm{Hz}^{-1}$ (i.e., $49.81 \pm 6.73 \%$ ) at $6: 00$ p.m.), while the medium-frequency band increased $\left(4.79 \pm 1.69 \mathrm{~mm} \cdot \mathrm{Hz}^{-1}\right.$ (i.e., $27.8 \pm 5.45 \%$ ) at $6: 00$ a.m. versus $6.13 \pm 1.83 \mathrm{~mm}^{2} \cdot \mathrm{Hz}^{-1}$ (i.e., $32.34 \pm 4.99 \%$ ) at 6:00 p.m.). In contrast, the contribution of the high-frequency band $\left(2.80 \pm 1.30 \mathrm{~mm}^{2} \cdot \mathrm{Hz}^{-1}\right.$ (i.e., $16.08 \pm 4.49 \%$ ) at $6: 00$ a.m. versus $3.40 \pm 1.14 \mathrm{~mm}^{2} \cdot \mathrm{Hz}^{-1}$ (i.e., $17.87 \pm 3.40 \%)$ at $6: 00$ p.m.) remained stable throughout the day (Figure 1(a)).

In the mediolateral direction, the statistical analysis of the fast Fourier transform revealed no significant difference ( $t=-0.61$; NS) in the total spectral energy between 6:00 a.m. $\left(12.09 \pm 3.91 \mathrm{~mm}^{2} \cdot \mathrm{Hz}^{-1}\right)$ and 6:00 p.m. (12.61 \pm $\left.3.49 \mathrm{~mm}^{2} \cdot \mathrm{Hz}^{-1}\right)$. As in the anteroposterior direction, the contribution of the low-frequency band (55.2\%) was higher than that of the medium-frequency $(28.6 \%)$ and high-frequency bands $(16.2 \%)$, regardless of the time-of-day $\left(F_{(2,36)}=83.41\right.$;
$P<0.001)$. More interestingly, the contribution of the various sensory inputs was modified throughout the day $\left(F_{(2,36)}=23.6 ; P<0.01\right)$. The proportion of the low-frequency band, which mostly accounts for visuovestibular regulation, was higher at $6: 00$ a.m. $\left(7.18 \pm 2.58 \mathrm{~mm}^{2} \cdot \mathrm{Hz}^{-1}\right.$ (i.e., $59.35 \pm$ $8.32 \%))$ than at $6: 00$ p.m. $\left(6.47 \pm 2.12 \mathrm{~mm}^{2} \cdot \mathrm{Hz}^{-1}\right.$ (i.e., $51.01 \pm$ $10.12 \%)$ ). In contrast, the contribution of the mediumfrequency band, considered to be an expression of cerebellar regulation, increased from $6: 00$ a.m. $\left(3.13 \pm 1.29 \mathrm{~mm} \cdot \mathrm{Hz}^{-1}\right.$ (i.e., $25.71 \pm 6.56 \%)$ ) to $6: 00$ p.m. $\left(3.99 \pm 1.51 \mathrm{~mm}^{2} \cdot \mathrm{Hz}^{-1}\right.$ (i.e., $31.55 \pm 7.79 \%)$ ). Finally, the contribution of the highfrequency band, which is sensitive to the involvement of the reflexive loop, remained stable throughout the day (1.79 \pm $0.64 \mathrm{~mm}^{2} \cdot \mathrm{Hz}^{-1}$ (i.e., $14.93 \pm 3.01 \%$ ) at $6: 00$ a.m. versus $2.20 \pm$ $0.74 \mathrm{~mm}^{2} \cdot \mathrm{Hz}^{-1}$ (i.e., $17.44 \pm 3.55 \%$ ) at 6:00 p.m.) (Figure $1(\mathrm{~b})$ ).

\section{Discussion}

In this study, oral temperature, vigilance, and postural sway parameters have been recorded in parallel to specify (i) the link between these parameters and (ii) the role played by vestibular, visual, and somatosensory inputs in the diurnal fluctuations of postural control. The results confirm the presence of diurnal fluctuations in postural control in relation to the increase in body temperature and sleepiness/vigilance levels improvement throughout the day. These diurnal fluctuations in postural control are mainly determined by a decrease in visuovestibular and an increase in cerebellar regulations throughout the day.

The evaluation of postural control during quiet standing indicates a significant effect of time-of-day on COP surface area and LFS ratio. These results are in agreement with those of a preliminary study [7], and they also confirm the idea that postural swaying is greater in the early morning, when the level of sleepiness is higher $[6,22]$ and body temperature is lower than in the evening $[4,22,23]$. The increase in the 

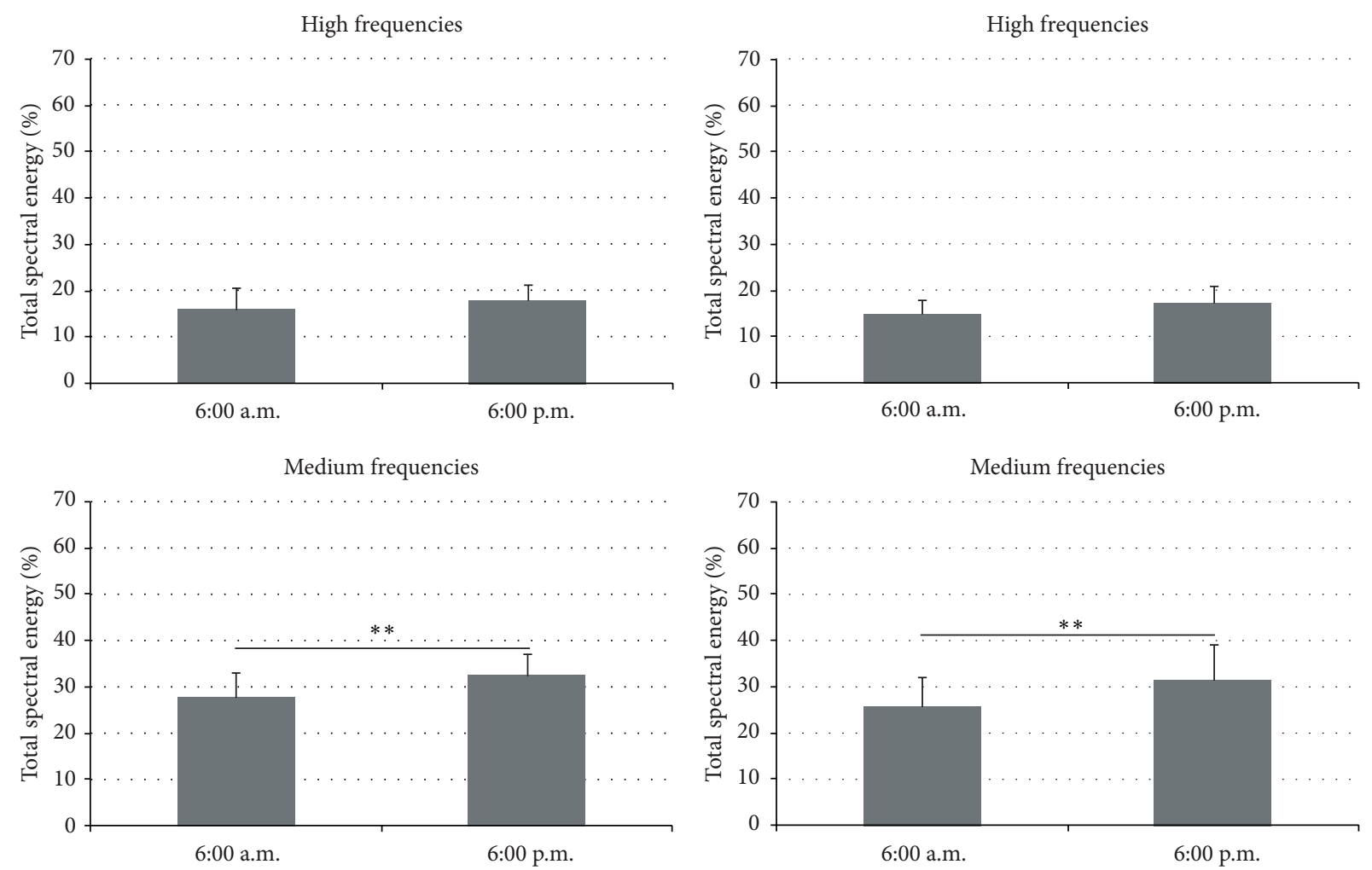

Low frequencies
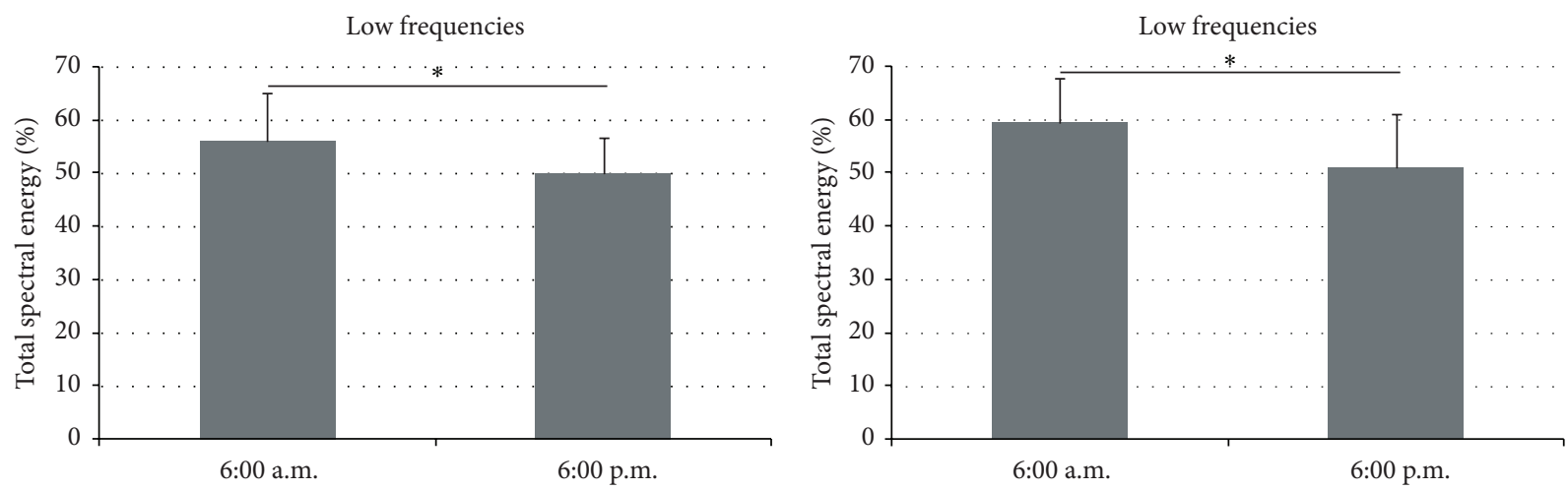

(a)

(b)

FIGURE 1: Percentage of total spectral energy in the anteroposterior direction (a) and mediolateral direction (b) measured at 6:00 a.m. and 6:00 p.m. Top: high-frequency band. Middle: medium-frequency band. Bottom: low-frequency band. ${ }^{* *}$ indicates a significant difference $(P<0.01) ;{ }^{*} P<0.05$.

LFS ratio in the evening is induced by a significant increase in $\mathrm{PL}$ and, in particular, in the mediolateral PL observed under the EO condition. These results were also obtained in previous studies $[3,23]$, in which lower COP velocities have been reported during morning sessions compared to evening ones after a night of normal sleep. As suggested by Santarcangelo et al. [47], these observations might be due to changes in postural strategies; they argue that a same or even reduced area swept in different conditions; larger LFS values indicate a longer COP trajectory and, thus, a greater number of shorter oscillations. According to a simple model of postural control, increased system stiffness and damping lead to decreases in sway displacement and increases in sway velocity [48]. Changes in postural control strategies during quiet stance involve changes in ankle, hip, or a combination of both $[49,50]$, which can be observed in postural recordings. An ankle strategy is mainly implicated in regulations in the anteroposterior direction and based on somesthetic input, while a hip strategy contributes to the stabilisation in the mediolateral direction and involves vestibular input [49]. Applying spectral analysis on each direction would bring further information on the sensory inputs underlying these changes in postural strategies.

Considering time-of-day effects on postural sway, the fact that the RI was statistically unchanged in the morning and in the evening confirms that the contribution of visual 
input is not modified by the hour of the recordings $[3,7]$. Therefore, the modifications observed in balance strategies throughout the day are probably due to modifications in the effectiveness of the other sensory systems (vestibular and/or proprioceptive) [51] and/or in the integration of the various sensory inputs via the cerebellum [52]. The decrease during the day in the low-frequency band [53] confirms that the visuovestibular system is highly affected by circadian rhythms and that the sensitivity of visual [54] and especially of vestibular [55] systems may improve throughout the day. As hypothesised by Morad et al. [4], the main submechanisms of postural control may have different thresholds and vulnerabilities to fatigue and sleep deprivation and also to circadian rhythmicity. In fact, our results suggest that the detection thresholds of imbalance situations may be more sensitive in the evening than in the morning. Previous studies have shown strong links between the mechanisms responsible for circadian rhythmicity and the receptors of the vestibular macula [56]. Moreover, the increase in fluid and blood circulation velocity at the cerebral level and, more precisely, in the vestibular system throughout the day [57] acts in parallel with the increase in body temperature [21], which may also contribute to the improvement of sensitivity of the vestibular system. As we observed in this study, this could induce more COP displacements on a reduced surface area (corrective movements). In contrast with the decrease observed in the low-frequency band, the contribution of the medium-frequency band, related to cerebellar regulation [45], was higher in both the anteroposterior and mediolateral directions at 6:00 p.m. than at 6:00 a.m. It seems that cerebellar regulation improves throughout the day in order to regulate postural sway more efficiently, as it requires faster adaptations. Several recent studies have shown that the "hip strategy" is particularly efficient in the maintenance of the centre of body mass (COM) above the support area [58], while minimising muscular and also neural activation [59]. This mechanism may be due to changes in CNS activation $[22,26]$, as reflected by the results obtained for the sign cancellation test, showing that the subjects were less sleepy in the evening. As for the involvement of the reflexive loop reflected by the high-frequency band $[4,53]$, our results confirmed those of previous studies reporting diurnal fluctuations in low- and medium-frequency bands with no modifications to the high-frequency band $[4,6]$. Since this higher part of the spectrum is the least involved in postural control, it seems that even though nervous conduction velocity [60] and muscular strength [61] are higher around 6:00 p.m. than at 6:00 a.m., the CNS does not ensure compensatory processes by using proprioceptive regulation [3].

Given the evolution of the different parameters recorded in this study, various assumptions may be formulated to explain changes in postural strategies between morning and evening measurements (Figure 2).

Firstly, various studies have demonstrated that motor spontaneous tempo increases throughout the day [62], which can also be observed in freely chosen pedal rate $[63,64]$. It can be proposed that the cerebellum, considered to be a cerebral structure responsible for movement coordination, may impulse a faster oscillatory frequency throughout the

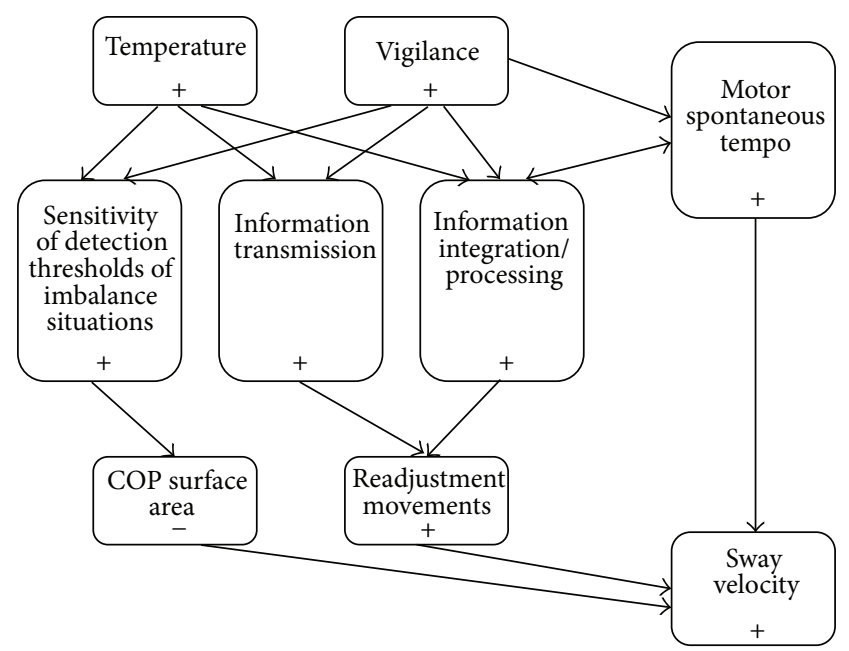

Figure 2: Processes involved in changes in postural strategies between morning and evening measurements. COP surface area: center-of-pressure surface area; +: 6:00 a.m. < 6:00 p.m.; -: 6:00 a.m. $>$ 6:00 p.m.

day. Secondly, nervous conduction [60] and the sensitivity of detection thresholds of near-fall situations may be improved in the evening, which may explain the decrease in surface area when temperature and vigilance levels are higher. Thirdly, as the reflexive loop is the least involved in postural regulation and muscular strength is not required to ensure balance, the CNS would not focus on this path to improve stability. All these observations coincide with the distribution of postural sway frequencies, indicating an increase in cerebellum regulation, probably due to an improvement of the sensitivity of detection thresholds of near-fall situations. This increase is in contrast with a disengagement of the visuovestibular contribution.

Although our work addresses an interesting question by examining which postural control mechanisms underlie the observed diurnal fluctuations, several issues should be considered before firm conclusions can be drawn. Firstly, only two time schedules have been selected to observe the diurnal fluctuation of postural control. However, even if more time points would have given precise information on the relationship between postural control and temperature and/or vigilance, the times of testing (6:00 a.m. and 6:00 p.m.) were chosen close to the expected bathyphase and acrophase and to allow the observation of maximal diurnal fluctuations [7]. In addition, various precautions were respected to limit potential confounding variables when studying time-of-day effects on postural control [5]. Secondly, the subjects spent 6 hours in bed, which could be considered as not sufficient to recover completely. Depending on the study under consideration, 6 to 9 hours are recommended [65]. Moreover, this experimental methodology is extensively used in chronobiological studies [32], and it has been confirmed that only one night with an early awakening does not influence the observation of diurnal fluctuations [66]. Thirdly, the subjects were awoken one hour before testing, which allowed sleep inertia effects to dissipate [36]. It has also been shown that 
many cognitive, psychomotor, and physical performances do not differ significantly between measurements carried out at 6:00 a.m. after being awakened either at 4:00 a.m. or at 5:00 a.m., that is, 1 or 2 hours before the test session $[33,34]$.

To conclude, the results of this study confirm that postural control is more effective in the evening, during the acrophase of the body temperature rhythm, and also when subjects feel less sleepy and more vigilant than they do in the early morning. Different strategies of postural control occur depending on time-of-day. It seems that in the morning there are fewer but extended COP displacements, whereas in the evening there are more short-length COP displacements in a reduced surface. Analysis of spectral content of postural sway bidirectionally indicates that these adaptations are probably induced by a decrease in visuovestibular regulation, in contrast with an improvement of cerebellar regulation, which are dependent on both the increase in body temperature and CNS activation. These results may also have direct application in terms of rehabilitation. Practitioners should focus on imbalanced situations with a higher frequency of readjustments in the evening to induce significant progresses.

\section{Conflict of Interests}

The authors declare that there is no conflict of interests regarding the publication of this paper.

\section{Acknowledgments}

This work was supported in part by a PREDIT-GO4 contract. Clément Bougard was granted his Ph.D. thesis by the Conseil Régional de Basse-Normandie (Regional Council of Lower Normandy) and the Institut National de Recherche sur les Transports et leur Sécurité (The French National Institute for Transport and Safety Research).

\section{References}

[1] R. Kohen-Raz, M. Himmelfarb, S. Tzur, A. Kohen-Raz, and Y. Shub, "An initial evaluation of work fatigue and circadian changes as assessed by multiplate posturography," Perceptual and Motor Skills, vol. 84, no. 2, pp. 547-557, 1996.

[2] N. Avni, I. Avni, E. Barenboim et al., "Brief posturographic test as an indicator of fatigue," Psychiatry and Clinical Neurosciences, vol. 60, no. 3, pp. 340-346, 2006.

[3] P. A. Gribble, W. S. Tucker, and P. A. White, "Time-of-day influences on static and dynamic postural control," Journal of Athletic Training, vol. 42, no. 1, pp. 35-41, 2007.

[4] Y. Morad, B. Azaria, I. Avni et al., "Posturography as an indicator of fatigue due to sleep deprivation," Aviation Space and Environmental Medicine, vol. 78, no. 9, pp. 859-863, 2007.

[5] T. Deschamps, J. Magnard, and C. Cornu, "Postural control as a function of time-of-day: influence of a prior strenuous running exercise or demanding sustained-attention task," Journal of NeuroEngineering and Rehabilitation, vol. 10, no. 1, article 26, 2013.

[6] Y. Liu, S. Higuchi, and Y. Motohashi, "Changes in postural sway during a period of sustained wakefulness in male adults," Occupational Medicine, vol. 51, no. 8, pp. 490-495, 2001.
[7] C. Bougard, M. Lepelley, and D. Davenne, "The influences of time-of-day and sleep deprivation on postural control," Experimental Brain Research, vol. 209, no. 1, pp. 109-115, 2011.

[8] M. S. Redfern, L. Yardley, and A. M. Bronstein, "Visual influences on balance," Journal of Anxiety Disorders, vol. 15, no. 1-2, pp. 81-94, 2001.

[9] L. M. Nashner, "Strategies for organization of human posture," in Vestibular and Visual Control on Posture and Locomotor Equilibrium, M. Igarashi and F. O. Black, Eds., pp. 1-8, Karger, Basel, Switzerland, 1985.

[10] K. A. Coffman, R. P. Dum, and P. L. Strick, "Cerebellar vermis is a target of projections from the motor areas in the cerebral cortex," Proceedings of the National Academy of Sciences of the United States of America, vol. 108, no. 38, pp. 16068-16073, 2011.

[11] R. C. Miall and E. W. Jenkinson, "Functional imaging of changes in cerebellar activity related to learning during a novel eye-hand tracking task," Experimental Brain Research, vol. 166, no. 2, pp. 170-183, 2005.

[12] P. L. Strick, R. P. Dum, and J. A. Fiez, "Cerebellum and nonmotor function," Annual Review of Neuroscience, vol. 32, pp. 413-434, 2009.

[13] M. P. Walker, R. Stickgold, D. Alsop, N. Gaab, and G. Schlaug, "Sleep-dependent motor memory plasticity in the human brain," Neuroscience, vol. 133, no. 4, pp. 911-917, 2005.

[14] L. Ayalon, S. Ancoli-Israel, Z. Klemfuss, M. D. Shalauta, and S. P. A. Drummond, "Increased brain activation during verbal learning in obstructive sleep apnea," NeuroImage, vol. 31, no. 4, pp. 1817-1825, 2006.

[15] E. Golomer, J. Crémieux, P. Dupui, B. Isableu, and T. Ohlmann, "Visual contribution to self-induced body sway frequencies and visual perception of male professional dancers," Neuroscience Letters, vol. 267, no. 3, pp. 189-192, 1999.

[16] E. Nagy, A. Feher-Kiss, M. Barnai, A. Domján-Preszner, L. Angyan, and G. Horvath, "Postural control in elderly subjects participating in balance training," European Journal of Applied Physiology, vol. 100, no. 1, pp. 97-104, 2007.

[17] M. Patel, S. Gomez, S. Berg et al., "Effects of 24-h and 36-h sleep deprivation on human postural control and adaptation," Experimental Brain Research, vol. 185, no. 2, pp. 165-173, 2008.

[18] B. L. Day, M. J. Steiger, P. D. Thompson, and C. D. Marsden, "Effect of vision and stance width on human body motion when standing: implications for afferent control of lateral sway," Journal of Physiology, vol. 469, pp. 479-499, 1993.

[19] Z. Kapoula, Q. Yang, T. Lê et al., "Medio-lateral postural instability in subjects with tinnitus," Frontiers in Neurology, vol. 2, Article ID Article 35, p. 35, 2011.

[20] N. B. Singh, W. R. Taylor, M. L. Madigan, and M. A. Nussbaum, "The spectral content of postural sway during quiet stance: influences of age, vision and somatosensory inputs," Journal of Electromyography and Kinesiology, vol. 22, no. 1, pp. 131-136, 2012.

[21] T. Reilly, "Human circadian rhythms and exercise," Critical Reviews in Biomedical Engineering, vol. 18, no. 3, pp. 165-180, 1990.

[22] T. Nakano, K. Araki, A. Michimori, H. Inbe, H. Hagiwara, and E. Koyama, "Nineteen-hour variation of postural sway, alertness and rectal temperature during sleep deprivation," Psychiatry and Clinical Neurosciences, vol. 55, no. 3, pp. 277-278, 2001.

[23] P. A. Gribble and J. Hertel, "Changes in postural control during a 48-hr. sleep deprivation period," Perceptual and Motor Skills, vol. 99, no. 3, pp. 1035-1045, 2004. 
[24] D. Weinert and J. Waterhouse, "The circadian rhythm of core temperature: effects of physical activity and aging," Physiology and Behavior, vol. 90, no. 2-3, pp. 246-256, 2007.

[25] D. G. M. Beersma and M. C. M. Gordijn, "Circadian control of the sleep-wake cycle," Physiology and Behavior, vol. 90, no. 2-3, pp. 190-195, 2007.

[26] A. Schlesinger, M. S. Redfern, R. E. Dahl, and J. R. Jennings, "Postural control, attention and sleep deprivation," NeuroReport, vol. 9, no. 1, pp. 49-52, 1998.

[27] M. Fabbri, M. Martoni, M. J. Esposito, G. Brighetti, and V. Natale, "Postural control after a night without sleep," Neuropsychologia, vol. 44, no. 12, pp. 2520-2525, 2006.

[28] S. S. Smith, T. Cheng, and G. K. Kerr, "The effect of extended wake on postural control in young adults," Experimental Brain Research, vol. 221, no. 3, pp. 329-335, 2012.

[29] M. W. Johns, "A new method for measuring daytime sleepiness: the Epworth sleepiness scale," Sleep, vol. 14, no. 6, pp. 540-545, 1991.

[30] J. A. Horne and O. Ostberg, "A self assessment questionnaire to determine morningness-eveningness in human circadian rhythms," International Journal of Chronobiology, vol. 4, no. 2, pp. 97-110, 1976.

[31] G. A. Kerkhof and H. P. van Dongen, "Morning-type and evening-type individuals differ in the phase position of their endogenous circadian oscillator," Neuroscience Letters, vol. 218, no. 3, pp. 153-156, 1996.

[32] T. Reilly and E. Bambaeichi, "Methodological issues in studies of rhythms in human performance," Biological Rhythm Research, vol. 34, no. 4, pp. 321-336, 2003.

[33] C. Bougard, N. Bessot, S. Moussay, B. Sesboüé, and A. Gauthier, "Effects of waking time and breakfast intake prior to evaluation of physical performance in the early morning," Chronobiology International, vol. 26, no. 2, pp. 307-323, 2009.

[34] C. Bougard, S. Moussay, A. Gauthier, S. Espié, and D. Davenne, "Effects of waking time and breakfast intake prior to evaluation of psychomotor performance in the early morning," Chronobiology International, vol. 26, no. 2, pp. 324-336, 2009.

[35] M. H. Bonnet and D. L. Arand, "Clinical effects of sleep fragmentation versus sleep deprivation," Sleep Medicine Reviews, vol. 7, no. 4, pp. 297-310, 2003.

[36] P. Tassi and A. Muzet, "Sleep inertia," Sleep Medicine Reviews, vol. 4, no. 4, pp. 341-353, 2000.

[37] B. Drust, J. Waterhouse, G. Atkinson, B. Edwards, and T. Reilly, "Circadian rhythms in sports performance-an update," Chronobiology International, vol. 22, no. 1, pp. 21-44, 2005.

[38] N. Souissi, N. Bessot, K. Chamari, A. Gauthier, B. Sesboüé, and D. Davenne, "Effect of time of day on aerobic contribution to the 30-s Wingate test performance," Chronobiology International, vol. 24, no. 4, pp. 739-748, 2007.

[39] R. Zazzo, "Le test des deux barrages," in Manuel pour L'examen Psychologique de L'enfant, R. Zazzo, Ed., pp. 470-575, 3rd edition, 1969.

[40] M. Casagrande, C. Violani, G. Curcio, and M. Bertini, "Assessing vigilance through a brief pencil and paper letter cancellation task (LCT): effects of one night of sleep deprivation and of the time of day," Ergonomics, vol. 40, no. 6, pp. 613-630, 1997.

[41] O. Caron, T. Gelat, P. Rougier, and J.-. Blanchi, "A comparative analysis of the center of gravity and center of pressure trajectory path lengths in standing posture: an estimation of active stiffness," Journal of Applied Biomechanics, vol. 16, no. 3, pp. 234247, 2000.
[42] P. M. Gagey and B. Weber, Posturologie; Régulation et dérèglements de la station debout, Masson, Paris, France, 1999.

[43] C. J. Njiokiktjien and J. A. P. Van Parys, "Romberg's sign expressed in a quotient. II. Pathology," Agressologie, vol. 17, pp. 19-24, 1976.

[44] E. Golomer, P. Dupui, and P. Bessou, "Spectral frequency analysis of dynamic balance in healthy and injured athletes," Archives Internationales de Physiologie, de Biochimie et de Biophysique, vol. 102, no. 3, pp. 225-229, 1994.

[45] T. Paillard, F. Noé, T. Rivière, V. Marion, R. Montoya, and P. Dupui, "Postural performance and strategy in the unipedal stance of soccer players at different levels of competition," Journal of Athletic Training, vol. 41, no. 2, pp. 172-176, 2006.

[46] T. Paillard, C. Costes-Salon, C. Lafont, and P. Dupui, "Are there differences in postural regulation according to the level of competition in judoists?" British Journal of Sports Medicine, vol. 36, no. 4, pp. 304-305, 2002.

[47] E. L. Santarcangelo, E. Scattina, G. Carli, A. Macerata, and D. Manzoni, "Hypnotizability-dependent modulation of postural control: effects of alteration of the visual and leg proprioceptive inputs," Experimental Brain Research, vol. 191, no. 3, pp. 331-340, 2008.

[48] C. Maurer and R. J. Peterka, "A new interpretation of spontaneous sway measures based on a simple model of human postural control," Journal of Neurophysiology, vol. 93, no. 1, pp. 189-200, 2005.

[49] F. B. Horak and L. M. Nashner, "Central programming of postural movements: adaptation to altered support-surface configurations," Journal of Neurophysiology, vol. 55, no. 6, pp. 1369-1381, 1986.

[50] R. Creath, T. Kiemel, F. Horak, R. Peterka, and J. Jeka, "A unified view of quiet and perturbed stance: simultaneous co-existing excitable modes," Neuroscience Letters, vol. 377, no. 2, pp. 75-80, 2005.

[51] K. M. Guskiewicz and D. H. Perrin, "Effect of orthotics on postural sway following inversion ankle sprain," Journal of Orthopaedic and Sports Physical Therapy, vol. 23, no. 5, pp. 326331, 1996.

[52] N. Teasdale and M. Simoneau, "Attentional demands for postural control: the effects of aging and sensory reintegration," Gait \& Posture, vol. 14, no. 3, pp. 203-210, 2001.

[53] E. Nagy, K. Toth, G. Janositz et al., "Postural control in athletes participating in an ironman triathlon," European Journal of Applied Physiology, vol. 92, no. 4-5, pp. 407-413, 2004.

[54] L. de Gennaro, M. Ferrara, G. Curcio, and M. Bertini, "Visual search performance across $40 \mathrm{~h}$ of continuous wakefulness: measures of speed and accuracy and relation with oculomotor performance," Physiology and Behavior, vol. 74, no. 1-2, pp. 197204, 2001.

[55] M. Wolf, I. E. Ashkenazi, and G. Leventon, "Circadian variation of nystagmus in healthy and sick subjects," Archives of Otolaryngology-Head and Neck Surgery, vol.116, no. 2, pp. 221223, 1990.

[56] P. M. Fuller, T. A. Jones, S. M. Jones, and C. A. Fuller, "Neurovestibular modulation of circadian and homeostatic regulation: vestibulohypothalamic connection?" Proceedings of the National Academy of Sciences of the United States of America, vol. 99, no. 24, pp. 15723-15728, 2002.

[57] M. A. B. Frey, T. H. Mader, J. P. Bagian, J. B. Charles, and R. T. Meehan, "Cerebral blood velocity and other cardiovascular responses to 2 days of head-down tilt," Journal of Applied Physiology, vol. 74, no. 1, pp. 319-325, 1993. 
[58] Y. Aramaki, D. Nozaki, K. Masani, T. Sato, K. Nakazawa, and H. Yano, "Reciprocal angular acceleration of the ankle and hip joints during quiet standing in humans," Experimental Brain Research, vol. 136, no. 1, pp. 463-473, 2001.

[59] A. D. Kuo and F. E. Zajac, "Human standing posture: Multijoint movement strategies based on biomechanical constraints," Progress in Brain Research, vol. 97, pp. 349-358, 1993.

[60] V. F. Ferrario, G. Tredici, and V. Crespi, "Circadian rhythm in human nerve conduction velocity," Chronobiologia, vol. 7, no. 2, pp. 205-209, 1980.

[61] G. Atkinson and T. Reilly, "Circadian variation in sports performance," Sports Medicine, vol. 21, no. 4, pp. 292-312, 1996.

[62] F. Dosseville, S. Moussay, J. Larue, A. Gauthier, and D. Davenne, "Physical exercise and time of day: influences on spontaneous motor tempo," Perceptual and Motor Skills, vol. 95, no. 3, pp. 965-972, 2002.

[63] S. Moussay, F. Dosseville, A. Gauthier, J. Larue, B. Sesboüe, and D. Davenne, "Circadian rhythms during cycling exercise and finger-tapping task," Chronobiology International, vol. 19, no. 6, pp. 1137-1149, 2002.

[64] S. Moussay, N. Bessot, A. Gauthier, J. Larue, B. Sesboüe, and D. Davenne, "Diurnal variations in cycling kinematics," Chronobiology International, vol. 20, no. 5, pp. 879-892, 2003.

[65] H. P. A. van Dongen, G. Maislin, J. M. Mullington, and D. F. Dinges, "The cumulative cost of additional wakefulness: dose-response effects on neurobehavioral functions and sleep physiology from chronic sleep restriction and total sleep deprivation," Sleep, vol. 26, no. 2, pp. 117-126, 2003.

[66] S. Banks and D. F. Dinges, "Behavioral and physiological consequences of sleep restriction," Journal of Clinical Sleep Medicine, vol. 3, no. 5, pp. 519-528, 2007. 

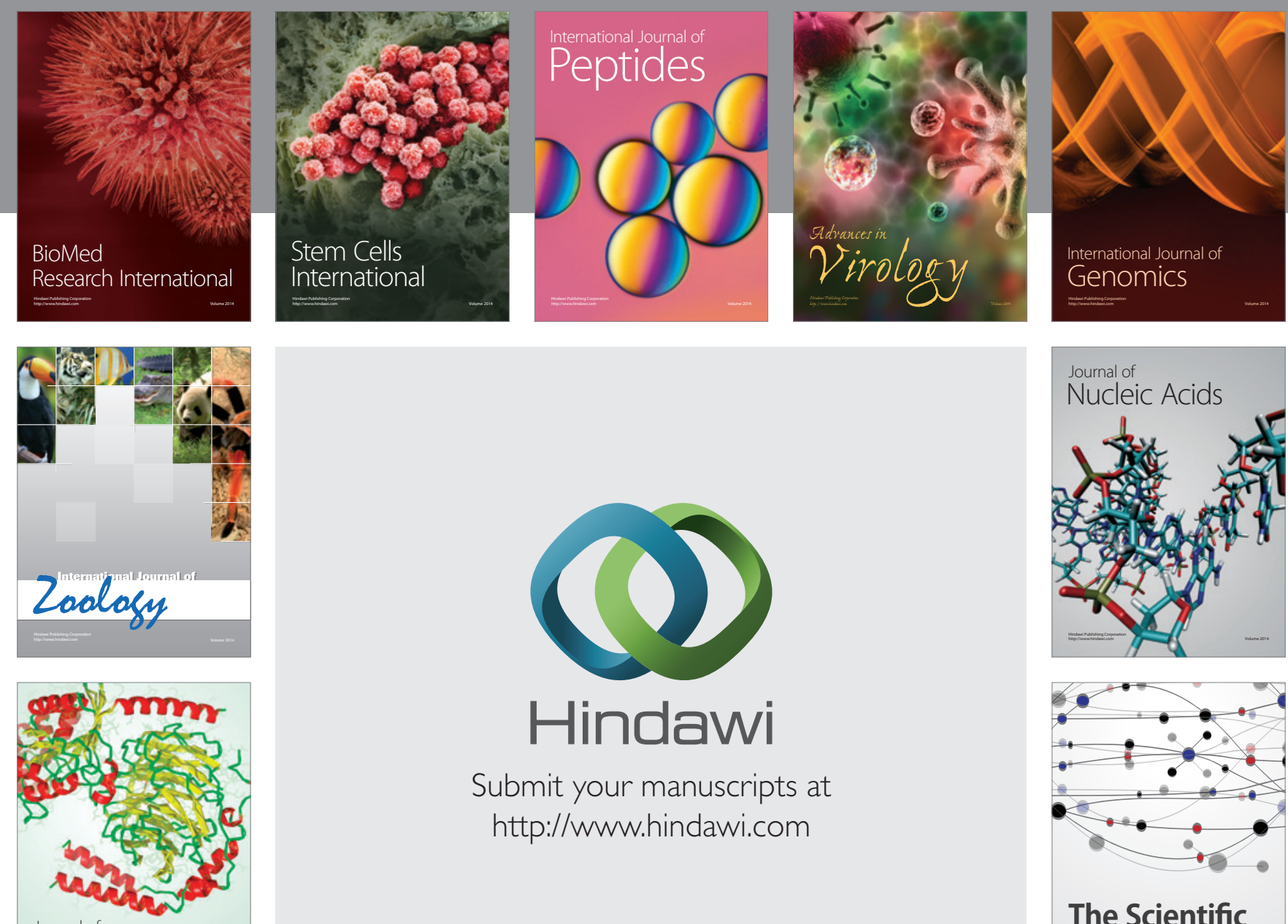

Submit your manuscripts at

http://www.hindawi.com

Journal of
Signal Transduction
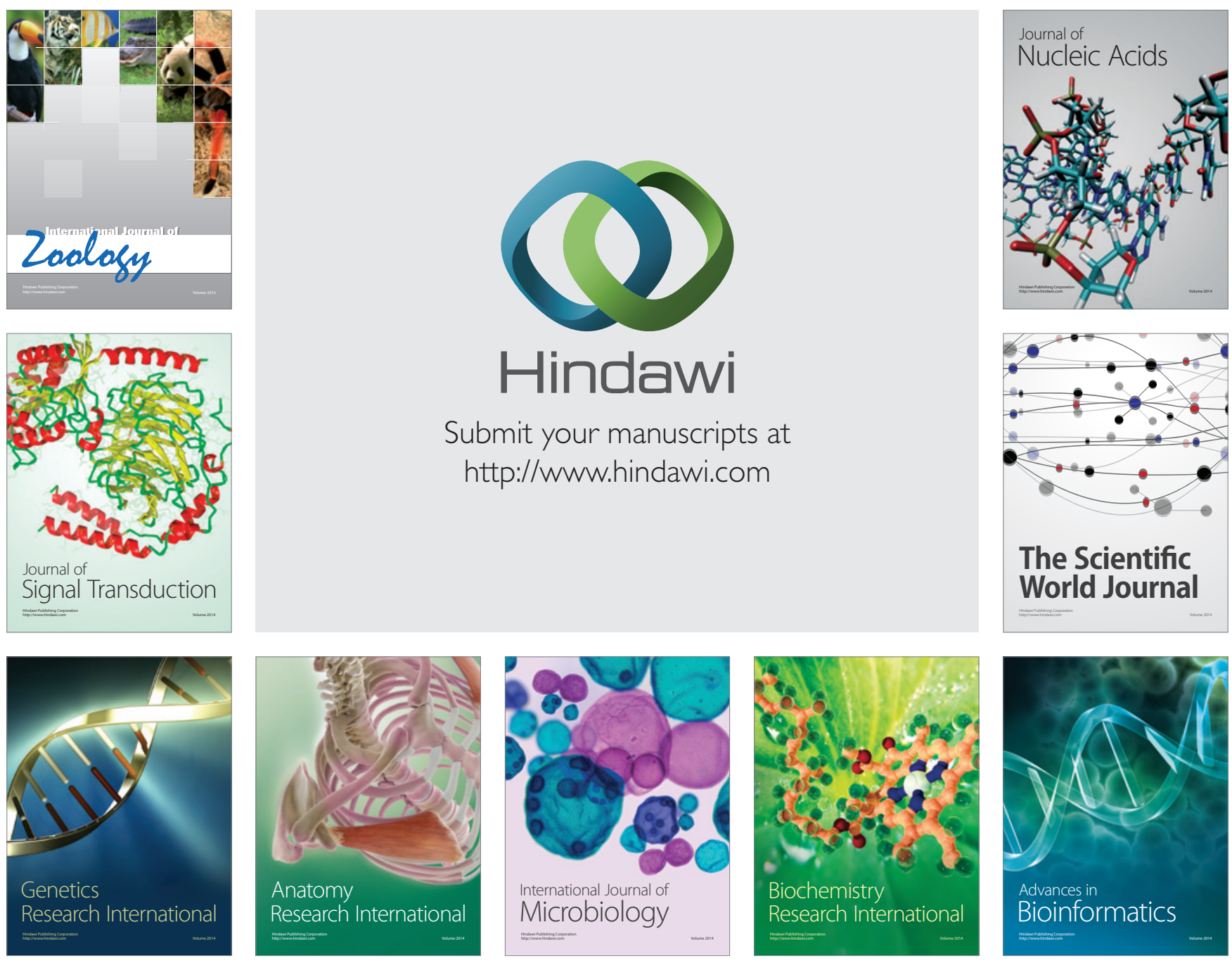

The Scientific World Journal
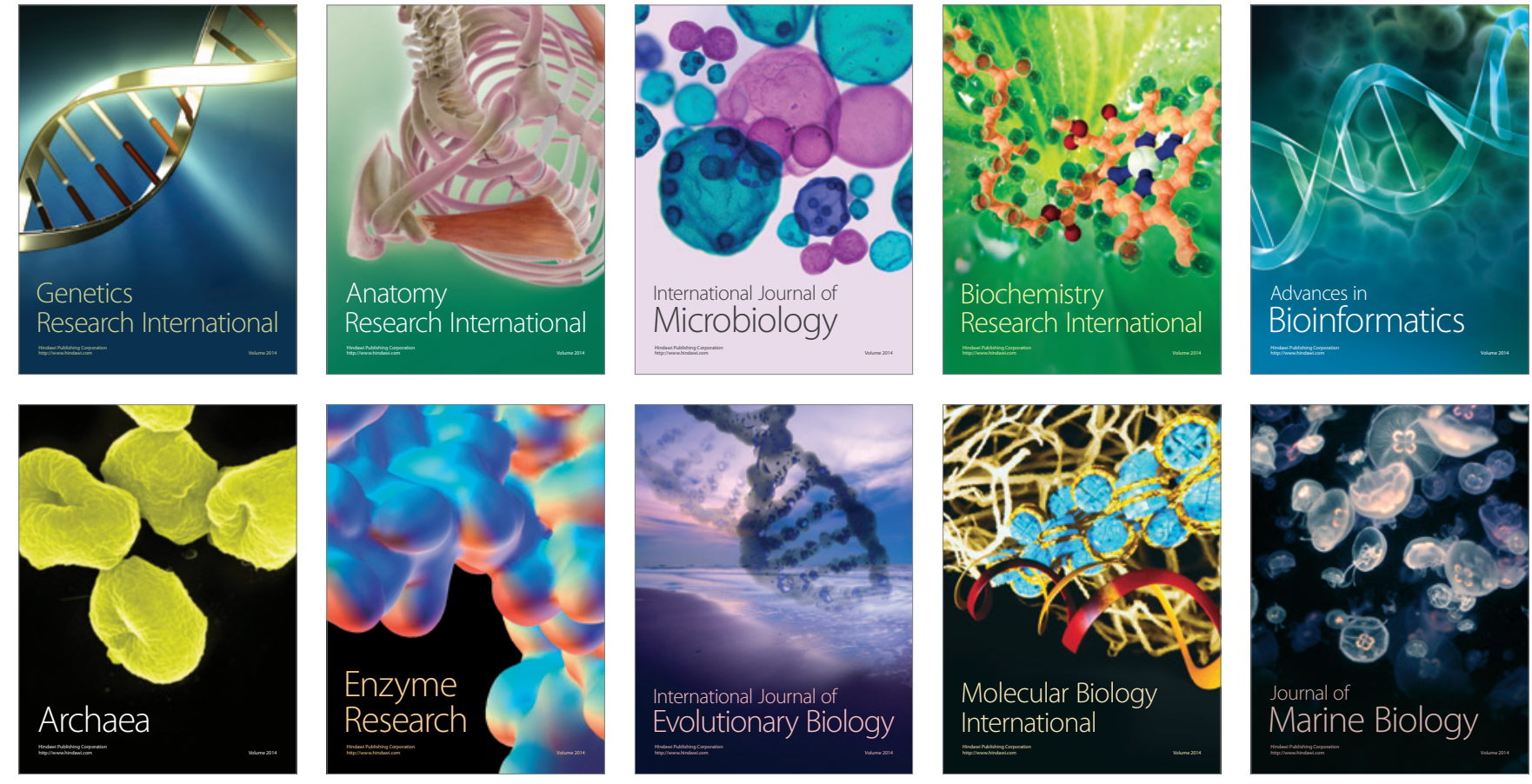